\title{
Understanding Patient Preferences and Unmet Needs in Non-alcoholic Steatohepatitis (NASH): Insights from a Qualitative Online Bulletin Board Study
}

\author{
Nigel S. Cook · Sarthak H. Nagar · Akanksha Jain · Maria-Magdalena Balp (D) • \\ Miriam Mayländer · Olivia Weiss · Satabdi Chatterjee
}

Received: October 1, 2018 / Published online: December 13, 2018

(C) The Author(s) 2018

\begin{abstract}
Introduction: The aim of this work was to understand how patients with non-alcoholic steatohepatitis (NASH) perceive their disease, unmet needs, and expectations regarding future treatment through online bulletin board (OBB) qualitative research.
\end{abstract}

Methods: OBB is an asynchronous online qualitative market research tool that provides an open forum for interactive discussion among participants. Patients with NASH were recruited via physician referral and completed a screener questionnaire to ensure their eligibility and willingness to participate. A trained moderator managed the discussion that allowed open answers and responses to other participants' posts. Patient responses were analyzed using a

Enhanced Digital Features To view enhanced digital features for this article go to https://doi.org/10.6084/ m9.figshare.7399241.

Electronic supplementary material The online version of this article (https://doi.org/10.1007/s12325018-0856-0) contains supplementary material, which is available to authorized users.

N. S. Cook $(\square) \cdot$ M.-M. Balp

Novartis Pharma AG, Basel, Switzerland

e-mail: nigel.cook@novartis.com

S. H. Nagar · A. Jain - S. Chatterjee

Novartis Healthcare Pvt Ltd, Hyderabad, India

M. Mayländer · O. Weiss

Gfk, Basel, Switzerland combination of different qualitative analytical tools.

Results: The OBB ran for 4 days and included 16 patients ( $n=8, \mathrm{UK} ; n=8$, US) with NASH (fibrosis stages F1-F3) and comorbidities including diabetes/prediabetes $(n=9)$ and obesity $(n=12)$. The key insights were (1) patients with NASH have a poor understanding of the disease, its progression, and management-they feel a lack of adequate educational support from their physicians; (2) diagnosis of NASH is incidental in most cases, mainly because patients fail to spontaneously associate their signs or symptoms with their liver condition; (3) comorbidities (obesity and diabetes) are more concerning to patients than NASH; and (4) patients perceive that NASH impacts their social life and work performance in more advanced stages.

Conclusions: This OBB provided valuable patient insights into NASH disease perception and management and revealed unmet need areas. In light of no approved therapies, these patient insights can inform early drug development strategies and stakeholder discussions on NASH.

Funding: Novartis Pharma AG, Basel.

Keywords: Disease education; NASH; Nonalcoholic steatohepatitis; OBB; Online bulletin board; Patient-based evidence; Patient perspective; Qualitative research; Unmet need 


\section{INTRODUCTION}

Non-alcoholic steatohepatitis, commonly known as NASH, is a progressive form of nonalcoholic fatty liver disease (NAFLD) that is characterized by excessive fat accumulation in the liver, inflammation, liver cell injury, and fibrosis [1]. NASH has been associated with an increased risk of morbidity and mortality from end-stage liver disease and hepatocellular carcinoma, especially in patients with advanced liver fibrosis [2]. In addition to clinical burden, NASH imposes a high economic burden on the healthcare system [3-7]. In the USA alone, an estimated 3-4 million patients with NASH and fibrosis, who are at the greatest risk for progression, account for an annual expenditure of US\$10-15 billion [7]. The high clinical and economic burden of NASH strongly indicates a need for effective treatment options. At present, there are no approved pharmacological therapies for NASH.

Patients with NASH experience a range of symptoms that negatively affect their quality of life including major depressive disorder, generalized anxiety disorder, fatigue, feeling bloated, having discomfort or pain around the liver, and feeling nauseous [8]. Data on overall disease burden in patients with NASH and information on patients' needs and preferences for NASH are scarce. Understanding patients' experiences and unmet needs in NASH is important in order to incorporate their perspectives in the development of new therapeutics.

Face-to-face focus group discussion has been a traditional approach to collect data on patients' experiences and perspectives on a disease condition [8-10]. With the availability of internet-based techniques, online focus group discussion is gaining popularity, particularly in the social and health sciences. The online bulletin board (OBB) is a moderated, asynchronous qualitative research tool that provides an open forum for virtual interactive focus group discussion on a topic among select participants [11]. A number of studies have shown the utility of online focus group technique and asynchronous platforms (e.g., email and discussion boards) to study various diseases and health- related issues in different health conditions [12-15]. In the present study, we used an OBB approach to understand how patients with NASH perceive their disease and what their unmet needs and expectations are regarding future treatment for NASH.

\section{METHODS}

Before conducting the OBB study, we performed a literature review to understand the current level of knowledge about NASH in published literature. Questions for the OBB were formulated after the literature review.

\section{Recruitment}

Patients with a medical diagnosis of NASH and mild (stage F1) to severe (F3) fibrosis (as per the METAVIR system of classification [16]) were eligible for the OBB forum conducted for four consecutive days in November 2016 (two parallel OBBs; one each in the USA and the UK). Patients were recruited via physician referral after the treating physician confirmed the diagnosis of NASH and patient eligibility. Physicians also recorded patients' NASH medical history and disease stage. Patients with cirrhosis (stage F4) or hepatocellular carcinoma were excluded. Recruited patients were invited to participate on an opt-in basis and received an honorarium at fair market value for their participation.

This study was conducted within the BHBIA/ ABPI (British Healthcare Business Intelligence Association/The Association of the British Pharmaceutical Industry) guidelines following the code of conduct of the Market Research Society and EphMRA. All procedures involving human participants were performed in accordance with the 1964 Helsinki declaration and its later amendments or comparable ethical standards. Ethics committee review was not sought for this online patient survey research. Informed consent was obtained electronically from all individual participants included in the study. Participants' rights and privacy were protected at all times throughout the study. Participants were granted the right to withdraw 
from the study at any time during the study conduct and to withhold information as they saw fit. All information/data that could identify respondents to third parties was kept strictly confidential; all respondents remained anonymous as their answers were reported in aggregate with the answers of all other participants.

\section{Procedure}

Participants were requested to login to the OBB at least twice a day for $30 \mathrm{~min}$ each and required to use self-chosen nicknames to maintain anonymity of discussion, which was structured by a trained moderator. The moderator posted questions pertaining to each day's theme on the OBB for participants to respond to. Participants were encouraged to comment on each other's posts and exchange experiences and insights. Following the fourth day of the OBB, all participants were invited to a 1.5 -h follow-up telephonic discussion (one each with the US and UK participants) to address any remaining questions and provide feedback on the OBB.

\section{Questions}

The questions asked on each day are presented in Appendix 1 (electronic supplementary material). The type of questions asked included mandatory questions, masked questions, openended questions, close-ended questions, and polls. Additionally, participants were invited to upload images illustrating their feelings on the disease. Overall, during the 4 days of the OBB forum, the study investigators sought to:

- Identify the medical journey that a typical patient with NASH goes through.

- Explore the patient's psychological and emotional attitude toward NASH.

- Understand the burden of NASH on the patient's daily living, finances, and employment.

- Assess physician-patient interaction regarding NASH, including quality of the relationship, emotions during consultations, and the patient's assessment of physician attitude toward NASH.
- Identify disease management approaches and patient expectations regarding potential treatments for NASH.

- Evaluate current unmet needs, expectations, acceptable risk, and value drivers of future treatment options.

- Solicit guidance on the optimal expected treatment outcome for short- and long-term disease management in NASH.

\section{Qualitative Analysis of Patient Responses}

A combination of different qualitative analytical tools (content analysis, grounded analysis, and discourse analysis) was used to analyze patients' responses [17-19]. For themes or questions that were predefined, content analysis was performed to assess emerging patterns. The patient inputs obtained were coded and analyzed in detail. Responses that could not be fit into the predefined themes were analyzed using grounded analysis, which helped in identifying areas for further exploration. Lastly, discourse analysis was conducted to comprehensively understand the context of patient responses and what stated information actually meant to them; the OBB approach provided an opportunity to follow up with either individual participants or the group as a whole in this regard. All responses were consolidated and reported under the respective theme of each day's discussion: (1) patient knowledge and perception, (2) patient insights regarding NASH diagnosis, monitoring, and management, (3) patient-reported information on NASH symptoms, comorbidities, and their impact on patient life, (4a) key unmet needs in NASH, and (4b) patient perspective on the effectiveness of lifestyle changes vs prescription medications and value drivers of future treatment options.

\section{RESULTS}

\section{Participant Characteristics}

Owing to the anonymous nature of the OBB, sociodemographic and background data were limited to those provided by the patients in the 
screening process and then posted spontaneously in the forum. In total, 16 patients (US, $n=8$ and $\mathrm{UK}, n=8$; female, $n=10$; electronic supplementary material, Appendix 2) diagnosed with NASH and fibrosis stages F1 to F3 were recruited. Of the eight patients from the USA, three each were Caucasian and Hispanic/Latino, and two were African American. Eight of the patients had previously undergone liver biopsy to confirm their NASH diagnosis (US, $n=5$ and UK, $n=3$ ). Patients who had not previously undergone biopsy as a confirmatory test, had been diagnosed by blood test, ultrasound, computed tomography (CT), and/or magnetic resonance imaging (MRI). Obesity (12 patients; US, $n=7$ and UK, $n=5$ ) and diabetes or prediabetic condition (9; US, $n=6$ and UK, $n=3$ ) were the most common comorbidities.

\section{Qualitative Patient Insights from OBB}

\section{Patient Knowledge and Perceptions of NASH}

Table 1 presents the insights obtained from patients regarding their knowledge and perception of the disease. Patients perceived that their physicians do not educate them adequately on the disease characteristics of NASH; therefore, most of them possess very basic understanding of NASH. A few patients had a good understanding of the disease, which was attributed either to education by their physician or self-learning through the internet. Around $50 \%$ of the patients were not aware of the current level of severity of their disease; a similar proportion suspected that NASH could progress to a life-threatening state.

About $50 \%$ of the participating patients did not acknowledge that NASH is a serious

Table 1 Disease knowledge and patient perception

\begin{tabular}{l}
$\begin{array}{l}\text { Emerging } \\
\text { pattern }\end{array}$ \\
\hline $\begin{array}{l}\text { Pack of } \\
\text { awareness }\end{array}$ \\
"I have lived with it all my life, I can manage it." (UK patient, fibrosis stage F1) \\
disease as something more serious." (UK patient, fibrosis stage F1) \\
"Many have it and do not even notice." (UK patient) \\
"I am always worried about my long-term health [...] the future is uncertain and I think the condition \\
will cut my life short." (US patient, fibrosis stage F2) \\
"Sometimes can't sleep as worried about my future health." (UK patient) \\
"I certainly do not know if there is anything I should be doing right now and if I am in any danger health \\
wise at present due to this condition." (UK patient) \\
"I wonder if I need a transplant one day. That scares me." (US patient, fibrosis stage F2) \\
"The imminent progression of it to permanent liver damage petrifies me." (US patient, fibrosis stage F3) \\
"Biopsy, appointments, hospitals, feeling scared." (UK patient) \\
"I guess the biggest challenge is always having in the back of my mind that my condition can worsen. That \\
my liver might eventually be the cause of my death." (US patient) \\
"The idea that I caused it myself is very sobering, it creeps up on you." (UK patient) \\
"I have never been a drinker, it's amazing I should have a liver condition [...] I feel sorry for myself." (UK \\
patient) \\
"Can be reversed by healthy lifestyle." (UK patient, fibrosis stage F1) \\
Gifestyle-related
\end{tabular}

$O B B$ online bulletin board 
disease-potentially because of lack of knowledge and, particularly in the UK, poor education through their treating physicians. While some patients associated NASH with an uncertain future that was characterized by a decline in health, fear, and need for medical interventions, others felt sorry for themselves, especially as they never consumed alcohol, but still suffered from a liver condition. A majority of patients felt that NASH is self-inflicted, mainly as a result of their (previous or current) unhealthy lifestyle and associated overweight, and consequently expressed guilt and shame for their condition.

\section{NASH Diagnosis, Monitoring, and Management}

In most cases, the diagnosis of NASH was incidental (e.g., during a routine health check or checkup for comorbidity), largely because patients did not have any signs or symptoms that they could associate with their liver condition. Although liver biopsy is recognized as a confirmatory diagnostic test for NASH, patients were afraid of the pain associated with biopsy, and hence reluctant to undergo this procedure.

Only a few patients felt comfortable and satisfied with the level of education and support received from their consulting physicians (Table 2). Other patients perceived that the treating physicians did not comprehensively educate them about treatment and management options for NASH-some patients even reported that their physicians did not seem to take NASH seriously or played it down. The advice from physicians to exercise more and eat more healthy was perceived as downplaying the importance of the condition by placing it in a similar category as being overweight (which most of the participants were anyway). No specific advice pertaining to their liver condition was thus retained from these consultations. This was found in particular to be an issue with patients being managed for their liver condition by their general physicians. In the USA, more patients were monitored by specialists and underwent frequent diagnostic testing compared to patients in the UK. Most patients wished that they would have received more education on NASH and its management and progression following diagnosis.

In terms of management strategies, most patients received the recommendation to lose weight and adopt a healthier lifestyle-often without concrete advice or further support. Most patients tried to follow the advice, but the biggest challenge for patients was to have the willpower and discipline to adhere to dietary and lifestyle changes. While in the USA, NASH patients were monitored by specialists regularly, in the UK this was true for only half of the patients (monitoring occurred between once every 3 months to once a year), and the type of examinations performed varied (typically, blood tests were performed, and in some cases ultrasound). A majority of patients expressed interest in having a treatment for NASH and were generally willing to take a systemic treatment if the physician recommended it or if there was evidence that their disease was progressing.

\section{Living with NASH-Symptoms, Comorbidities, and Impact on Patients' Life}

Figure 1 presents patients' insights into symptoms associated with NASH, comorbidities, and the impact of NASH on patients' life. Patients did not spontaneously report any symptoms that they could directly associate with NASH. However, after probing, they acknowledged experiencing the following symptoms (though not necessarily linking it to NASH): fatigue, weakness or lethargy, depression and anxiety, itching, abdominal pain or discomfort, and sleeping problems. Of these, fatigue or daytime tiredness was the most commonly reported symptom that affected the patients most in their daily life (Table 3).

Patients suffered from multiple comorbidities that they perceived to be more concerning than NASH, with obesity (12 of total 16 participants) and type 2 diabetes (9 of 16 participants) being the most common. Most of the participants were overweight. Patients attributed their overweight to be the cause of NASH and believed that weight loss was the key to resolve their liver condition. Several patients reported that NASH does not have a significant impact on their daily life, particularly those with less- 
Table 2 Patient insights regarding diagnosis, monitoring, and disease management

\begin{tabular}{ll}
\hline Emerging pattern & Patient quotes \\
\hline $\begin{array}{l}\text { Embarrassment at NASH } \\
\text { diagnosis }\end{array}$ & "I felt embarrassed because I had an unhealthy lifestyle-I put on a lot of weight." (UK \\
patient) & "I felt embarrassed that this happened to me because I felt it is my fault." (US patient) \\
Perceived lack of physician & "I was concerned of course and confused as to why my doctor wasn't doing more." (US \\
support & patient, fibrosis stage F1) \\
"I felt let down that they expect you to go away and be ok with no information as they say & fatty liver is common." (UK patient) \\
"Someone sitting me down and talking me through what I should and shouldn't do, like \\
caring support to give me hope, that's all I want." (UK patient) \\
"Doctors should not wait until the condition gets to stage 3 to start taking it seriously." (US \\
patient) \\
"I really think I'm missing medical support. No one has really explained my prognosis or any \\
treatment options. I am kind of in the dark." (US patient) \\
"Better education would be good, guidance to eating habits and foods to help improve or \\
reduce the issue. I have been taking the pills long enough now not to need reminding. I \\
think mainly support from GP or specialist." (UK patient) \\
"A NASH information pack would be nice, also with plenty of information about the drug \\
and how it works." (UK patient) \\
"Detailed information about NASH, how to care for your liver, dietary info, supplement info, \\
who to contact if you need support. I would like a physical copy, but also an online format." \\
(UK patient) \\
"What is missing is a(n) educational course designed for those with NASH because I have had \\
to do a lot of research on my own to seek and gather knowledge about NASH. I would like \\
a(n) educational DVD designed for people dealing with NASH." (US patient)
\end{tabular}

NASH non-alcoholic steatohepatitis

severe stages of the disease; however, NASH impacted social life in those with advanced stages of the disease (Table 3 ).

Patients perceived that NASH did impact their work performance. Work performance was considered to decline as the disease progressed, although they found it difficult to determine if this was due to the progression of NASH or other conditions. Fatigue, in particular, was perceived to prevent patients from working full time, going to the office, or completing their normal work duties. Patients with a more severe disease stage mentioned taking frequent time off work due to medical appointments, ultimately leading to job changes. A need for "significant" support with cooking, cleaning, and personal hygiene was also mentioned, because of patients' inability to stand for long periods.

"The areas of my life that have become troublesome include adherence to appointment schedule and changing eating habits. I have to take time off of work and adjust my diet. I must use sick days to take off. I must use a recipe planner to eat better. This is all very inconveniencing." (US, male) 


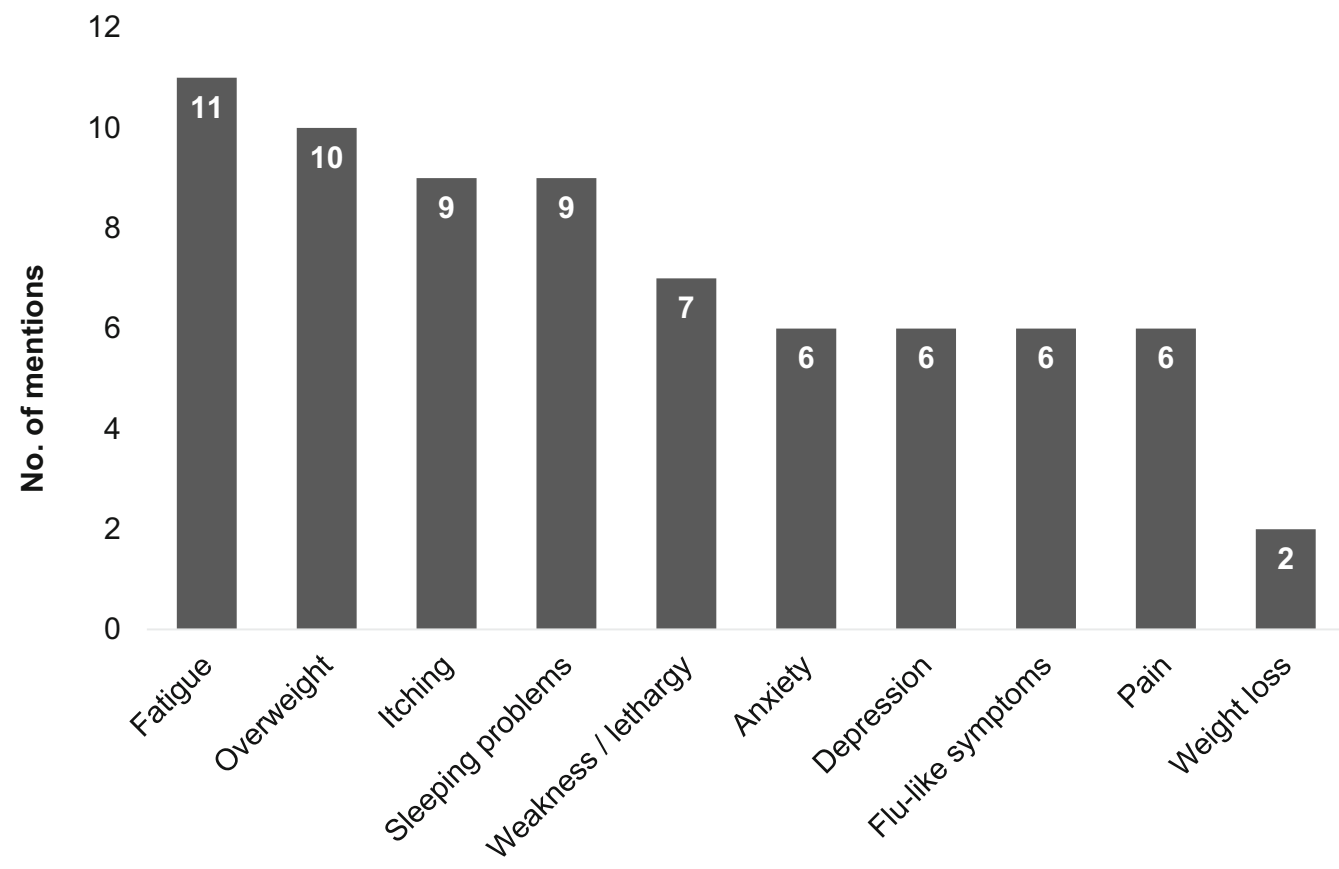

Fig. 1 Symptoms mentioned by patients

"I cannot do heavy labor anymore and had to be shifted to a back office position." (US patient)

Across different severity stages and income groups, half of the US participants and one UK patient felt that NASH imposed a financial burden due to their limited ability to work.

When patients were asked to upload pictures illustrating their feelings on the disease, most of these pictures reflected emotions of being sad, down, frustrated, sorry for themselves, and bewildered. Only a few patients managed to stay positive and expressed pride in being able to do so.

\section{Key Unmet Needs in NASH}

When asked about the biggest unmet need areas in managing their current liver condition, patients commonly pointed out the lack of cure, lack of education on the disease and its management, followed by not knowing their disease prognosis (Table 4). Upon further probing with regards to the unmet need experienced in daily life and expectations for additional support, patients overall agreed that there is minimal updated information on NASH; therefore, a more in-depth treatment plan and information concerning ways to improve or stabilize their liver condition were requested. Consistent with their responses on NASH diagnosis and management, patients highlighted the need for continuous support from their treating physicians to understand the course of treatment and ways to reverse their liver damage. Some patients also mentioned that they felt the need for a constant support system to counsel them on new symptoms experienced and the steps that can be undertaken to improve overall well-being (Table 4).

\section{Desired Properties of Future Treatments}

Patients provided mixed responses to questions about their views on medical treatment options versus lifestyle changes. While some patients favored lifestyle changes, thus avoiding potential side effects of a medical treatment, others mentioned that they have faith in potential drug treatment (13 of the 16 participating patients were willing to take a drug treatment for NASH), as they felt this could work faster and better than lifestyle modifications which had failed them in the past. Patients also acknowledged that physicians advised them to adopt a healthier lifestyle. Thus, patients 
Table 3 Impact of NASH on patients' life

\begin{tabular}{l} 
Emerging \\
pattern \\
\hline $\begin{array}{l}\text { Pymptoms } \\
\text { (fatigue) }\end{array}$ \\
"It hasn't really affected my life [...] the only impact is that I get very tired all the time." (UK patient) \\
"I notice that I tire more from doing less." (US patient) \\
"I miss a lot of things my kids are into, because I am just too tired." (US patient) \\
"I didn't get diagnosed until I stopped working. I was always tired which could have been a sign that I \\
wasn't aware of." (UK patient) \\
"Before the diagnosis I ate what I wanted, partied and drank, so socially it's been a massive change." (UK \\
"Patient) \\
"After the diagnosis I felt really helpless, until I made the decision to change my eating habits. When I \\
changed my eating habits I did not want to go out because I couldn't eat like everybody else." (US \\
patient) \\
"I feel as if I have not the energy to take on a full-time job, but I don't know whether this is due to my \\
liver condition or my MS or just being a busy mom." (UK patient) \\
"I cannot do heavy labor anymore and had to be shifted to a back office position." (US patient) \\
"I notice that I get tired during the day even after having a full night's sleep. It affects the tasks I can get \\
done during the day." (US patient) \\
"I work for myself, so it does not impact my working life. I plan my schedule around doctor's \\
appointments." (US patient) \\
"There is no impact on my day to day job but when I had a flare-ups before diagnosis I thought it was flu \\
and had time off as I felt awful." (UK patient)
\end{tabular}

$M S$ multiple sclerosis, $O B B$ online bulletin board

believed that by adopting a healthier lifestyle, they could reverse their condition. Regardless, they said that they found it hard to stick to a weight loss or exercise regime or healthier eating habits (Table 4).

When asked about the ideal outcomes expected from future treatment(s) in NASH, reversal of liver damage (within a year) emerged as the key request, along with improved quality of life (within a year), prevention of disease progression (within a year), and reduction of symptoms such as feeling less tired (within months). Among the long-term outcomes (within 5-10 years), patients stated avoidance of organ transplant and prevention of disease progression as their key desired outcomes from treatment (Table 4).

\section{DISCUSSION}

Patient involvement has become an essential element in providing more informed and transparent decision-making in health technology assessments [20], clinical guidelines development [21], healthcare priority setting [22], and healthcare research [23, 24]. To our knowledge, this is the first patient needs and preferences study conducted among those living with NASH. The study findings provide unique insights into patients' perspectives and their views on the disease, its impact, and their expectations for future treatment. In the absence of approved therapies for NASH, insights derived from patients through this OBB, particularly about future treatment 
Table 4 Patient perspective on unmet needs in NASH and desired properties of future treatment

Emerging pattern
Lack of treatment as a key unmet need
Mixed opinion toward medical treatment vs
lifestyle intervention

\section{Patient quote}

"My biggest unmet need is not knowing my prognosis." (US patient)

"It's just the dissatisfaction that there is no cure for liver disease yet." (US patient)

"My biggest unmet needs are dealing with fatigue and itching." (US patient)

"I would like to get my liver enzymes down as low as possible and that is my unmet need." (UK patient)

“The need for a cure really...!” (UK patient)

"If there was a drug that could maybe stem progression that would be good. But there is a danger that I would then rely on that... not change my lifestyle maybe?” (UK patient)

"I'd definitely prefer drugs. These lifestyle changes aren't [a] sure thing. I trust medicine.” (UK patient)

"I really feel I take enough medication and not having been a drinker I don't have to change my lifestyle due to NASH.” (UK patient)

"I take so many meds that adding more wouldn't phase me. I have already had to make major changes in my diet. Any more changes would be extremely difficult and unwelcome." (US patient)

"I prefer lifestyle changes because you don't have to worry about side effects.” (US patient)

"I prefer lifestyle alterations over prescription meds any day because with the drugs there may be long-term side effects that show up later in life. Drugs are quicker and can be targeted, but lifestyle changes overall are safer." (US patient)

Reversal of liver damage as an ideal outcome

"I would hope treatments could reduce or reverse the damage to my liver."

of future NASH treatment

(US patient)

"Number 1: Stopping of continual decay of the liver within a year. Number 2: Feeling overall better within a year. Number 3: Ruling out the possibility of an organ transplant within 5-10 years.” (US patient)

"1st: No more symptoms-within 6 months; 2nd: good test results-within 8 months; 3rd: high quality of life-within 12 months” (US patient)

"Less tired (within a few months)...no flu-like symptoms (within a few months)...no progression (over years).” (UK patient)

NASH non-alcoholic steatohepatitis

attributes, can be incorporated into early drug development and evidence collection strategies and can influence stakeholder discussions.
Our findings highlighted disease education as one of the key unmet needs. Patients with NASH have a very basic understanding of their disease, its progression, and management. 
However, patients fail to differentiate between symptoms related to NASH and those experienced as a result of comorbidities. For patients in our study, the comorbidities were more concerning than NASH itself as a "disease". This attitude can potentially be attributed to the low awareness and knowledge about the disease and to the lack of clear signs and symptoms felt by the patients that could be attributed by them to NASH specifically. Consequently, it is necessary to educate patients with NASH on their liver condition, diagnosis, and the progressive nature of the disease.

Another important finding from our study was that patients perceived a lack of support from physicians (especially general practitioners or primary care physicians) on various aspects of NASH, such as disease characteristics, prognosis, and advice on disease management goals. This indicates that, currently, patients feel left alone with their disease and strive for emotional support and medical education from their treating physicians. This could also be because the general practitioners do not have sufficient information on the disease or that patients do not listen to the treating physicians' advice (e.g., change of lifestyle, which is the only effective therapy currently, and the increased risk associated with not following a healthier lifestyle), or a mix of both. Therefore, it is important that physicians find ways to effectively communicate the seriousness of NASH as a progressive disease and provide regular checkups and adequate motivational support to their patients. A partial solution to this could also be review and consultation with dieticians or additional interventions such as smartphone applications engaging the patients in better management of their health [25]. It has been reported that familial history of diabetes is associated with NASH and fibrosis [26]. Thus, discussion around familial history could potentially be a strong motivator to encourage patients to look after their health better. Studies have attempted to evaluate the role of other healthcare professionals involved in healthcare delivery (e.g., nurse practitioner and physician assistant) for patient education in NASH and have indicated that such collaboration can play an important role in optimal patient outcomes $[27,28]$. Thus, educational programs designed to improve the dialogue between treating physician, healthcare professionals, and patients may help bridge the gap. Unavailability of treatment for NASH was identified as an important unmet need by patients. In the absence of any approved pharmacological treatment, the available treatment guidelines recommend lifestyle interventions as the first step of disease management for NASH $[29,30]$, with this also being advocated by treating physicians. From the patients' perspective, however, the fact that they are offered only "lifestyle modification" in the absence of symptoms and without any therapeutic option was interpreted as a lack of importance placed on the disease and "lack of physician guidance" on NASH management. Although existing evidence supports that as little as $5-10 \%$ weight loss can have an astonishing effect on the pathology of NASH [25], adapting to lifestyle modifications is difficult for most patients. In fact, even in a clinical study setting, only few patients managed to achieve a $10 \%$ weight loss [25]. Since most patients do not realize the impact of NASH (especially the early forms), it would be difficult for them to appreciate the true effect of a therapy. It will be important to use outcome measures during clinical development, which are relevant to patients and help them to recognize the value of a new therapy for themselves, especially in patients with comorbidities such as diabetes, obesity, or hyperlipidemia, where an increased risk for mortality and faster progression of liver disease has been shown [31]. Furthermore, as pointed out by Kennedy-Martin et al., it is important that novel treatments developed for NASH not only decrease disease progression but also reduce the impact on health-related quality of life of patients, with a focus on meaningful outcomes relevant to patients [32]. In addition, the use of such outcome measures will help in the discussion with other stakeholders, such as the health technology assessment bodies that increasingly focus on patientrelevant endpoints.

When asked for the symptoms of NASH, patients in the present OBB study did not seem 
to link some of the symptoms directly to their disease and only admitted after being prompted that fatigue and weakness or lethargy were very common; some also reported depression and anxiety, itching, abdominal pain or discomfort, and sleeping problems. Previous analyses of focus group discussions and interviews also reported a prevalence of fatigue among patients with NASH (67\%), as well as a similar range of other symptoms (abdominal malaise, nausea, dietary aversions and frustrations, and others) experienced by smaller subgroups of patients [32]. Such symptoms have a serious negative impact on quality of life, daily activities, and social behaviors of patients [33].

Confirmatory diagnosis by liver biopsy was performed in only $50 \%$ of the total recruited patients for the present OBB study. Although biopsy is considered as the gold standard for diagnosis of NASH, it is not routinely conducted for all patients because of its high costs, invasive nature, and associated procedural risks [34]. The lack of an available treatment option post-diagnosis is also a likely reason that physicians do not routinely subject patients to liver biopsy. In this scenario, developing alternative non-invasive techniques for diagnosis of NASH in patients could help in disease diagnosis and subsequently monitoring as and when future treatments become available. Besides, there are currently no practicable means available to the patients (liver biopsy could be a means, albeit most people are reluctant to commit to this regularly) or the physicians for monitoring disease progression or improvement [35]. This, in turn, initiates a "vicious cycle" of psychological conundrum: having no symptoms, no treatment, and no way to measure the course of the liver disease, and making patients feel even more helpless. These aspects need to be explored in detail through future research with physicians to help understand their perspectives regarding diagnosis, symptoms associated with NASH and comorbidities, physicians' motivations toward disease management strategies, and the communication between physicians and patients after the diagnosis of NASH.

We have successfully applied this OBB research methodology in other disease areas that include indications ranging from chronic obstructive pulmonary disease, obesity, to even very rare conditions like pigmented villonodular synovitis [36-39]. The strength of the present study methodology was the maintenance of patient anonymity, which led to openminded, spontaneous, and interactive responses from patients. The OBB forum enabled individual answers, vivid descriptions, and deep reflection in exercises as well as interaction between patients, all of which helped in understanding the more holistic impact of NASH directly from the patients' perspective. Importantly, the community form of interaction with "like" people in the same situation, together with the anonymity, encouraged people to share their feelings and emotions even on sensitive topics (such as appearance, hygiene, and their intimate relationships).

Certain study limitations should also be recognized. This was a short-term exploratory study to understand patients' experiences with NASH and assess their unmet needs and preferences for future treatment in NASH. Qualitative patient preference methodologies typically have small sample sizes and are a scientifically well-established and trusted method of gaining insights around a specific research topic $[40,41]$. Owing to the small number of patients in the OBB, the study findings may not be representative of the broader real-world NASH population; patients participating in this study, for example, required access to the internet, and are likely to have more interest in their disease than the broader population. The OBB included a mixed group of patients with or without different types of comorbidities. As a result of the small patient numbers, it was not possible to determine if there are any differences in the needs and perceptions between the subgroups of patients. Nevertheless, our study provides a sound basis and valuable insights to inform the design of future studies to investigate longer duration and quantitative analysis of patient perceptions from larger patient groups or specific subgroups. Further research to understand physician awareness, attitudes, and approaches to NASH management would complement the findings of the present study and may help improve physician-patient communication for better disease management. 


\section{CONCLUSIONS}

Learning from patients' perspectives will be essential to integrate their expectations into future therapies and management strategies for NASH. Patients with NASH have a poor understanding of the disease and the symptoms commonly associated with the condition. As a result of their lack of knowledge on symptoms, severity, and progression of the disease, patients with NASH find it difficult to differentiate between symptoms related to NASH and other health issues or comorbidities. The present study contributes to a growing body of literature concerning approaches to integrate the patient voice into early drug development strategies and stakeholder discussions on NASH. More research is required to align these patient perspectives alongside physician knowledge and perception of NASH.

\section{ACKNOWLEDGEMENTS}

We thank all the participants of the study.

Funding. Sponsorship for this study, Open Access fees, and article processing charges were funded by Novartis Pharma AG, Basel. All authors had full access to all of the data in this study and take complete responsibility for the integrity of the data and accuracy of the data analysis.

Medical Writing and Editorial Assistance. We thank Bhavik Shah, PhD of Novartis Healthcare Pvt Ltd, Hyderabad (India), funded by Novartis Pharma AG, Basel, for providing medical editorial assistance with this manuscript and Susann Walda (Gfk, Basel, Switzerland) for her support during conduct of the online bulletin board study.

Authorship. All named authors meet the International Committee of Medical Journal Editors (ICMJE) criteria for authorship for this article, take responsibility for the integrity of the work as a whole, and have given their approval for this version to be published.
Authorship Contributions. NSC conceptualized and designed the study, including the discussion guide, with inputs from $M M B, M M$, OW, SHN, and AJ. MM and OW moderated the online bulletin board during the study, with all authors contributing to the daily review of patient posts and input regarding follow-up questions. SC and NSC prepared the first draft of manuscript. All authors contributed to the interpretation of results and revision of subsequent drafts and reviewed and approved the final version before submission.

Prior Presentation. The findings of this study were partially presented at CADTH Symposium, Ottawa, Canada, April 23-25, 2017.

Disclosures. Nigel S. Cook is a full-time employee of and holds stocks in Novartis. Sarthak H. Nagar is a full-time employee of Novartis. Akanksha Jain is a full-time employee of Novartis. Maria-Magdalena Balp is a full-time employee of Novartis. Miriam Mayländer and Olivia Weiss (both were employee of GfK; effective October 10, 2018, GfK became part of the organization of Ipsos SA) declare no conflict of interest. Satabdi Chatterjee was an employee of Novartis Healthcare Pvt Ltd until June 29, 2018 and is now employed by the University of Houston within the Department of Pharmaceutical Outcomes \& Policy.

Compliance with Ethics Guidelines. All procedures performed in studies involving human participants were in accordance with the 1964 Helsinki declaration and its later amendments or comparable ethical standards. This study was conducted within the BHBIA/ ABPI guidelines following the code of conduct of the Market Research Society and EphMRA. Ethics committee review was not sought for this online patient survey research. Informed consent was obtained electronically from all individual participants included in the study. Participants' rights and privacy were protected at all times throughout the study. Participants were granted the right to withdraw from the study at any time during the study conduct and to withhold information as they saw fit. All information/data that could identify 
respondents to third parties was kept strictly confidential; all respondents remained anonymous as their answers were reported in aggregate with the answers of all other participants.

Data Availability. The datasets (online bulletin board transcripts) generated and analyzed during the current study are available from the corresponding author on reasonable request.

Open Access. This article is distributed under the terms of the Creative Commons Attribution-NonCommercial 4.0 International License (http://creativecommons.org/licenses/ by-nc/4.0/), which permits any noncommercial use, distribution, and reproduction in any medium, provided you give appropriate credit to the original author(s) and the source, provide a link to the Creative Commons license, and indicate if changes were made.

\section{REFERENCES}

1. Kopec KL, Burns D. Nonalcoholic fatty liver disease: a review of the spectrum of disease, diagnosis, and therapy. Nutr Clin Pract. 2011;26:565-76.

2. LaBrecque DR, Abbas Z, Anania F, et al. World Gastroenterology Organisation global guidelines: nonalcoholic fatty liver disease and nonalcoholic steatohepatitis. J Clin Gastroenterol. 2014;48:467-73.

3. Younossi ZM, Blissett D, Blissett R, et al. The economic and clinical burden of nonalcoholic fatty liver disease in the United States and Europe. Hepatology. 2016;64:1577-86.

4. Younossi ZM, Henry L. Economic and quality-oflife implications of non-alcoholic fatty liver disease. Pharmacoeconomics. 2015;33:1245-53.

5. Younossi ZM, Zheng L, Stepanova M, Henry L, Venkatesan C, Mishra A. Trends in outpatient resource utilizations and outcomes for Medicare beneficiaries with nonalcoholic fatty liver disease. J Clin Gastroenterol. 2015;49:222-7.

6. Sayiner M, Otgonsuren M, Cable R, et al. Variables associated with inpatient and outpatient resource utilization among Medicare beneficiaries with nonalcoholic fatty liver disease with or without cirrhosis. J Clin Gastroenterol. 2017;51:254-60.
7. Younossi ZM, Henry L, Bush H, Mishra A. Clinical and economic burden of nonalcoholic fatty liver disease and nonalcoholic steatohepatitis. Clin Liver Dis. 2018;22:1-10.

8. Kennedy-Martin T, Bae JP, Paczkowski R, Freeman E. Health-related quality of life burden of nonalcoholic steatohepatitis: a robust pragmatic literature review. J Patient Rep Outcomes. 2017;2:28.

9. Moser A, Korstjens I. Series: practical guidance to qualitative research. Part 3: Sampling, data collection and analysis. Eur J Gen Pract. 2018;24:9-18.

10. LaVela SL, Gallan A. Evaluation and measurement of patient experience. Patient Exp J. 2014;1:28-36.

11. Rolland SE, Parmentier G. The benefit of social media: bulletin board focus groups as a tool for cocreation. Int J Mark Res. 2013;55:809-27.

12. Griffiths KM, Reynolds J, Vassallo S. An online, moderated peer-to-peer support bulletin board for depression: user-perceived advantages and disadvantages. JMIR Ment Health. 2015;2:e14.

13. Schwarzer R, Satow L. Online intervention engagement predicts smoking cessation. Prev Med. 2012;55:233-6.

14. Ikunaga A, Nath SR, Skinner KA. Internet suicide in Japan: a qualitative content analysis of a suicide bulletin board. Transcult Psychiatry. 2013;50:280-302.

15. Thomas C, Wootten A, Robinson P. The experiences of gay and bisexual men diagnosed with prostate cancer: results from an online focus group. Eur J Cancer Care. 2013;22:522-9.

16. Bedossa P, Poynard T. An algorithm for the grading of activity in chronic hepatitis C. The METAVIR Cooperative Study Group. Hepatology. 1996;24:289-93.

17. Humphrey L, Willgoss T, Trigg A, et al. A comparison of three methods to generate a conceptual understanding of a disease based on the patients' perspective. J Patient Rep Outcomes. 2017;1:9.

18. Im E-O, Chee W. Practical guidelines for qualitative research using online forums. Comput Inform Nurs. 2012;30:604.

19. Noyes J, Hannes K, Booth A, et al. Qualitative and implementation evidence and Cochrane reviews. In: Cochrane handbook for systematic reviews of interventions. The Cochrane Collaboration, pp 1-26. Retrieved from http://qim.cochrane.org/ supplemental-handbook-guidance. Accessed 23 Sept 2018. 
20. Kinter ET, Schmeding A, Rudolph I, dosReis S, Bridges JF. Identifying patient-relevant endpoints among individuals with schizophrenia: an application of patient-centered health technology assessment. Int $\mathrm{J}$ Technol Assess Health Care. 2009;25:35-41.

21. van Kammen J, Jansen CW, Bonsel GJ, Kremer JA, Evers JL, Wladimiroff JW. Technology assessment and knowledge brokering: the case of assisted reproduction in the Netherlands. Int $\mathrm{J}$ Technol Assess Health Care. 2006;22:302-6.

22. van der Ham AJ, van Erp N, Broerse JE. Monitoring and evaluation of patient involvement in clinical practice guideline development: lessons from the Multidisciplinary Guideline for Employment and Severe Mental Illness, the Netherlands. Health Expect. 2016;19:471-82.

23. Boivin A, Lehoux P, Lacombe R, Burgers J, Grol R. Involving patients in setting priorities for healthcare improvement: a cluster randomized trial. Implement Sci. 2014;9:24.

24. Brett J, Staniszewska S, Mockford C, et al. A systematic review of the impact of patient and public involvement on service users, researchers and communities. Patient. 2014;7:387-95.

25. Vilar-Gomez E, Martinez-Perez Y, Calzadilla-Bertot $\mathrm{L}$, et al. Weight loss through lifestyle modification significantly reduces features of nonalcoholic steatohepatitis. Gastroenterology. 2015;149:36778.e5.

26. Satapathy SK, Sanyal AJ. Epidemiology and natural history of nonalcoholic fatty liver disease. Semin Liver Dis. 2015;35(03):221-35.

27. Werner KT, Perez ST. Role of nurse practitioners in the management of cirrhotic patients. J Nurse Pract. 2012;8(10):816-21.

28. Hearn C, Ellington BJ, Jones R. The role of the nurse practitioner in the management of nonalcoholic fatty liver disease. Gastroenterol Nurs. 2018;41(5):424-6.

29. Chalasani N, Younossi Z, Lavine JE, et al. The diagnosis and management of nonalcoholic fatty liver disease: practice guidance from the American Association for the Study of Liver Diseases. Hepatology. 2018;67:328-57.

30. National Guideline Centre (UK). Non-alcoholic fatty liver disease: assessment and management. London: National Institute for Health and Care Excellence (UK); 2016.
31. Byrne CD, Targher G. NAFLD: a multisystem disease. J Hepatol. 2015;62:S47-64.

32. Kennedy-Martin T, Bae JP, Paczkowski R, Freeman E. Health-related quality of life burden of nonalcoholic steatohepatitis: a robust pragmatic literature review. J Patient Rep Outcomes. 2017;2:28.

33. Palsgrove AHS, Ferguson B, Cheng R, Dombroski J, Cole JC. PRM130-Development of a conceptual framework for assessing disease-specific patient-reported outcomes in nonalcoholic steatohepatitis. In: Presented at the ISPOR 21st annual international meeting May 21-25, 2016; Washington Hilton, Washington, DC, USA

34. Ratziu V, Charlotte F, Heurtier A, et al. Sampling variability of liver biopsy in nonalcoholic fatty liver disease. Gastroenterology. 2005;128:1898-906.

35. Fedchuk L, Nascimbeni F, Pais R, et al. Performance and limitations of steatosis biomarkers in patients with nonalcoholic fatty liver disease. Aliment Pharmacol Ther. 2014;40:1209-22.

36. Landskroner K, Walda S, Weiss O, Pallapotu V, Cook N. VP161 identification of needs of pigmented villonodular synovitis patients using online bulletin board. Int J Technol Assess Health Care. 2017;33(S1):222-3.

37. Cook NS, Tripathi P, Weiss O, Walda S, George AT, Bushell A. Patient needs, perceptions and attitudinal drivers associated with obesity: a qualitative online bulletin board study. Poster presented at: ISPOR Europe [PSY209]; Nov 10-14, 2018; Barcelona, Spain.

38. Cook NS, Gey J, Oezel B, et al. Sleep disturbance and fatigue as consequences of cough and mucus production in chronic obstructive pulmonary disease: insights from a patient online bulletin board study. Poster presented at: ISPOR Europe [PRS98]; Nov 10-14, 2018; Barcelona, Spain.

39. Cook NS, Gey J, Oezel B, et al. Impact of cough and mucus on the emotional and psychosocial wellbeing in patients with chronic obstructive pulmonary disease: a qualitative patient online bulletin board study. Poster presented at: ISPOR Europe [PRS99]; Nov 10-14, 2018; Barcelona, Spain.

40. Hammarberg K, Kirkman M, De Lacey S. Qualitative research methods: when to use them and how to judge them. Hum Reprod. 2016;31(3):498-501.

41. Creswell JW, Creswell JD. Research design: qualitative, quantitative, and mixed methods approaches. Los Angeles: SAGE; 2018. 